University of Nebraska - Lincoln

DigitalCommons@University of Nebraska - Lincoln

Christian Binek Publications

Research Papers in Physics and Astronomy

August 2001

\title{
Exchange Bias in a generalized Meiklejohn-Bean approach
}

Christian Binek

University of Nebraska-Lincoln, cbinek@unl.edu

Follow this and additional works at: https://digitalcommons.unl.edu/physicsbinek

Part of the Physics Commons

Binek, Christian, "Exchange Bias in a generalized Meiklejohn-Bean approach" (2001). Christian Binek Publications. 40.

https://digitalcommons.unl.edu/physicsbinek/40

This Article is brought to you for free and open access by the Research Papers in Physics and Astronomy at DigitalCommons@University of Nebraska - Lincoln. It has been accepted for inclusion in Christian Binek Publications by an authorized administrator of DigitalCommons@University of Nebraska - Lincoln. 


\title{
Exchange bias in a generalized Meiklejohn-Bean approach
}

\author{
Ch. Binek*, A. Hochstrat, W. Kleemann \\ Laboratorium für Angewandte Physik, Gerhard-Mercator-Universität Duisburg, Lotharstr. 1, D-47048 Duisburg, Germany
}

Received 27 February 2001; received in revised form 23 May 2001

\begin{abstract}
A generalized Meiklejohn-Bean model is considered in order to derive an analytic expression for the dependence of the exchange bias field on the layer thickness involved in ferromagnetic/antiferromagnetic heterosystems, on the orientation of the applied magnetic field with respect to the magnetic easy axes and on the quenched magnetization $M_{\mathrm{AF}}$ of the antiferromagnetic pinning layer. While $M_{\mathrm{AF}}$ is a well-known feature of field-cooled dilute antiferromagnets, it seems to occur quite generally also in pure AF pinning substrates. The new analytic expressions are successfully compared with recent experimental results and Monte Carlo investigations. (C) 2001 Elsevier Science B.V. All rights reserved.
\end{abstract}

PACS: $75.70 . \mathrm{Cn} ; 75.10 . \mathrm{Hk}$

Keywords: Exchange bias; Interfaces; Ferromagnetism; Antiferromagnetism; Unidirectional anisotropy

\section{Introduction}

The Stoner-Wohlfarth model is a well-established phenomenological approach in order to describe coherent hysteretic magnetization reversal processes of single domain particles and magnetic thin films [1]. Although it does not take into account the influence of domain formation, its simplicity makes it a favorable first approach in order to interpret experimental data. E.g., it has been successfully applied in a phenomenological description of the exchange bias effect, which is observed in ferromagnetic (FM)/antiferromagnetic (AF) heterostructures. After field cooling of FM/ AF layered heterosystems to below the AF Néel

\footnotetext{
*Corresponding author. Fax: +49-203-379-1965.

E-mail address: binek@kleemann.uni-duisburg.de (C. Binek).
}

temperature, the hysteresis loop of the FM film is shifted along the magnetic field axis by the exchange bias field $\mu_{0} H_{\mathrm{e}}$. This shift reflects the unidirectional anisotropy, which originates from the FM/AF coupling at the interface. The coupling has been introduced into a phenomenological free energy expression by Meiklejohn and Bean (MB) $[2,3]$. The bilinear exchange between the FM/AF interface magnetic moments gives rise to the unidirectional anisotropy energy.

In the limit of infinite anisotropy of the antiferromagnet, the $\mathrm{MB}$ model yields the simple, but powerful formula $\mu_{0} H_{\mathrm{e}}=-J S_{\mathrm{AF}} S_{\mathrm{F}} /$ $\left(M_{\mathrm{FM}} t_{\mathrm{FM}}\right)$ [2,3]. It exhibits the well-known dependencies of $\mu_{0} H_{\mathrm{e}}$ on the FM layer thickness $t_{\mathrm{FM}}$ [4], on the magnetization of the FM layer [5] and on the interface magnetic moments $S_{\mathrm{AF}}$ and $S_{\mathrm{F}}$. No information about the origin of $S_{\mathrm{AF}}$ and $S_{\mathrm{FM}}$ is provided by the MB model. In 
addition, the coupling constant $J$ enters the MB approach only as a phenomenological constant. A lot of theoretical work, which tackles the microscopic foundation of these parameters or develops alternative descriptions of the undirectional anisotropy, has been done [6]. Nevertheless, the simple $\mathrm{MB}$ formula already points out the necessity of net magnetic moments at the interface, not only on the FM, but also on the AF side in order to obtain finite exchange bias. Without exception this holds also in the case of so-called compensated AF surfaces, where $S_{\mathrm{AF}} \neq 0$ requires deviations from the ideal $\mathrm{AF}$ order [7].

In the framework of the present analysis we point out the implications of the MB model on the $\mu_{0} H_{\mathrm{e}}$ vs. $t_{\mathrm{AF}}$ dependence which is, e.g., observed in experiments on $\mathrm{NiFe} / \mathrm{FeMn}$ heterostructures [8] and in Monte Carlo studies on Ising systems of FM and diluted AF layers [9] exhibited in Figs. 1 and 2, respectively. It turns out, that both non-vanishing AF magnetization $M_{\mathrm{AF}}$ and a finite angle $\theta$ between the applied magnetic field and the magnetic easy axes tune the details of the $t_{\mathrm{AF}}$ dependencies. We exploit the full capability of the MB approach in analytic expressions and overcome restrictions due to partial solutions accounting either for $\theta[10,11]$ or $t_{\mathrm{AF}}[12]$ alone.

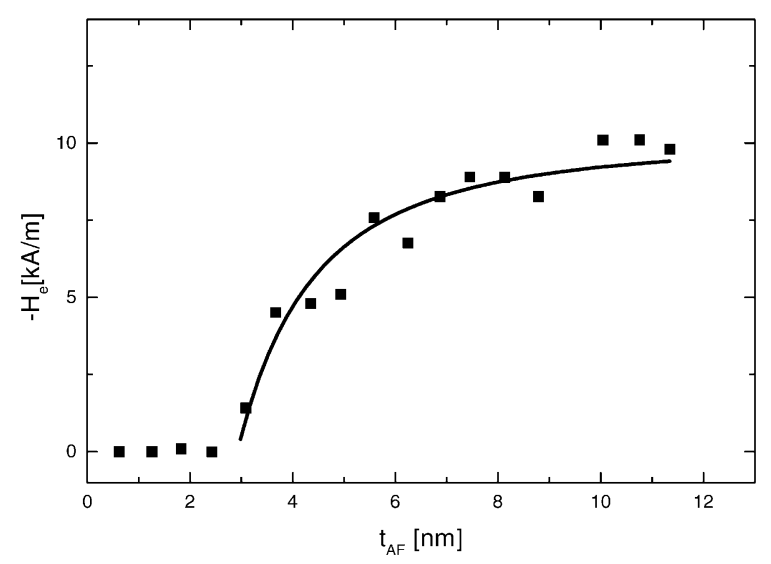

Fig. 1. Dependence, $H_{\mathrm{e}}$ vs. $t_{\mathrm{AF}}$, of the exchange bias field in $\mathrm{Ni}_{80} \mathrm{Fe}_{20} / \mathrm{FeMn}$ [8]. The line shows the best fit of Eq. (7) to the data at $t_{\mathrm{AF}}>3 \mathrm{~nm}$.

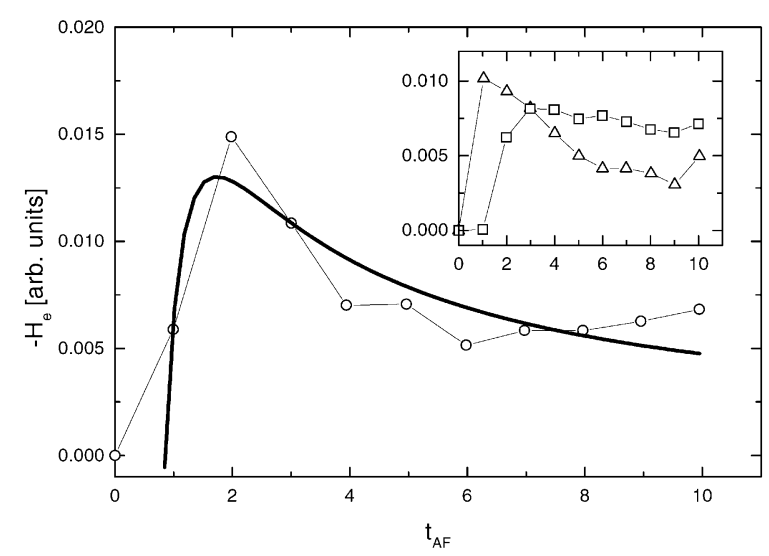

Fig. 2. Data from Monte Carlo simulations [9] of the exchange bias field as a function of the number of AF layer monolayers, $t_{\mathrm{AF}}$, are shown for $40 \%$ (circles), $30 \%$ (triangles in the inset), and $60 \%$ (squares in the inset) quenched site dilution of the AF. The bold solid line is a best fit of Eq. (9) to the data at $40 \%$ dilution.

\section{Model and analysis}

We start from the free energy per unit area $[2,3]$ completed by adding a Zeeman term involving $M_{\mathrm{AF}}$,

$$
\begin{aligned}
F= & -\mu_{0} H M_{\mathrm{FM}} t_{\mathrm{FM}} \cos (\theta-\beta) \\
& -\mu_{0} H M_{\mathrm{AF}} t_{\mathrm{AF}} \cos (\theta-\alpha) \\
& +K_{\mathrm{FM}} t_{\mathrm{FM}} \sin ^{2} \beta \\
& +K_{\mathrm{AF}} t_{\mathrm{AF}} \sin ^{2} \alpha-J S_{\mathrm{AF}} S_{\mathrm{FM}} \cos (\beta-\alpha) .
\end{aligned}
$$

Here $H$ is the applied magnetic field and $M_{\mathrm{FM} / \mathrm{AF}}$, $t_{\mathrm{FM} / \mathrm{AF}}, K_{\mathrm{FM} / \mathrm{AF}}, S_{\mathrm{FM} / \mathrm{AF}}$ are the absolute values of the magnetization, the layer thickness, the uniaxial anisotropy constant, and the interface magnetic moments of the FM/AF layer. The latter ones can be interpreted as macroscopic moments because the MB model assumes parallel orientation of all FM moments during the entire process of coherent rotation. Hence, the FM spins fulfill the condition $\underline{s}_{i}^{\mathrm{FM}}=\underline{s}^{\mathrm{FM}} \forall i$, and the interaction of the microscopic spins at the interface can be transformed into an interaction of the macroscopic interface moments according to $\sum_{i, j} \underline{S}_{i}^{\mathrm{FM}} \underline{\underline{S}}_{j}^{\mathrm{AF}} \propto \underline{S}_{\mathrm{FM}} \underline{S}_{\mathrm{AF}}$. They are coupled via $J$, the exchange interaction constant. $\theta, \beta$ and $\alpha$ are the angles between $H$, 
$M_{\mathrm{FM}}, M_{\mathrm{AF}}$ and the FM/AF anisotropy axis, which are aligned in parallel for simplicity (see Fig. 3).

The AF magnetization, $M_{\mathrm{AF}}$, is generally assumed to be zero. This is reasonable in the case, where the sublattice magnetizations mutually compensate in the long-range AF ordered state. However, this is no longer the case in diamagnetically diluted AF systems. They are known to decay into a random-field-induced domain state with frozen excess magnetization when cooling to below $T_{\mathrm{N}}$ in an external magnetic field [13]. This mechanism seems to control the appearance of interface magnetization, $S_{\mathrm{AF}} \neq 0$, at compensated AF surfaces and thus enables exchange bias [7,9]. On the other hand, also the excess bulk magnetization, $M_{\mathrm{AF}} \neq 0$, of a quenched AF domain state may be important by virtue of the corresponding Zeeman energy term in Eq. (1). Surprisingly, metastable domain states can also be induced in non-diluted AF pinning layers. They are probably due to interface roughness [14-17] and give rise to both $M_{\mathrm{AF}}$ and excess susceptibility.

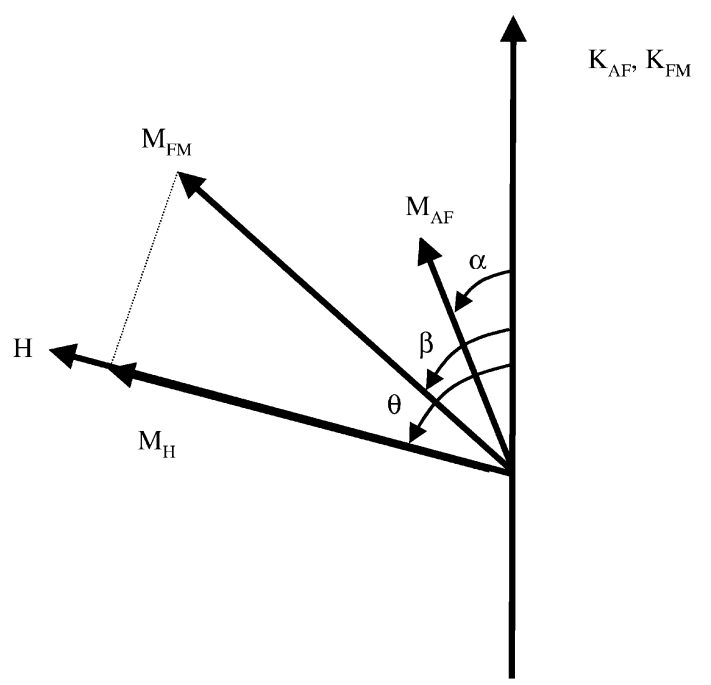

Fig. 3. Vector diagram involving the angles $\alpha, \beta$, and $\theta$ related to the orientation of the net $\mathrm{AF}$ magnetization $M_{\mathrm{AF}}$, the magnetization of the ferromagnet $M_{\mathrm{FM}}$ and the applied magnetic field $H$ with respect to the easy axis of the antiferroand ferromagnet designated by the corresponding anisotropy constants $K_{\mathrm{AF}}$ and $K_{\mathrm{FM}}$, respectively. $M_{H}$ indicates the projection of $M_{\mathrm{FM}}$ onto the field direction.
In the case of infinite anisotropy $K_{\mathrm{AF}}$, the minimization of the free energy yields $\alpha=0$. Hence, in the case of strong but finite anisotropy, a series expansion of Eq. (1) with respect to $\alpha=0$ is reasonable. It reads

$$
\begin{aligned}
F \approx & -J S_{\mathrm{AF}} S_{\mathrm{FM}} \cos \beta-\mu_{0} H M_{\mathrm{FM}} t_{\mathrm{FM}} \cos (\theta-\beta) \\
& -\mu_{0} H M_{\mathrm{AF}} t_{\mathrm{AF}} \cos \theta+K_{\mathrm{FM}} t_{\mathrm{FM}} \sin ^{2} \beta \\
& +\alpha\left(-J S_{\mathrm{AF}} S_{\mathrm{FM}} \sin \beta-\mu_{0} H M_{\mathrm{AF}} t_{\mathrm{AF}} \sin \theta\right) \\
& +\alpha^{2}\left(K_{\mathrm{AF}} t_{\mathrm{AF}}+\frac{1}{2} J S_{\mathrm{AF}} S_{\mathrm{FM}} \cos \beta\right. \\
& \left.+\frac{1}{2} \mu_{0} H M_{\mathrm{AF}} t_{\mathrm{AF}} \cos \theta\right) .
\end{aligned}
$$

This expression is minimized with respect to $\beta$ and $\alpha$, which is physically equivalent to the determination of the equilibrium angles $\beta_{\mathrm{eq}}$ and $\alpha_{\mathrm{eq}}$ of vanishing torque. $\partial F / \partial \alpha=0$ yields

$$
\alpha_{\mathrm{eq}}^{\alpha}=\frac{J S_{\mathrm{AF}} S_{\mathrm{FM}} \sin \beta+\mu_{0} H M_{\mathrm{AF}} t_{\mathrm{AF}} \sin \theta}{2 K_{\mathrm{AF}} t_{\mathrm{AF}}+J S_{\mathrm{AF}} S_{\mathrm{FM}} \cos \beta+\mu_{0} H M_{\mathrm{AF}} t_{\mathrm{AF}} \cos \theta}
$$

From $\partial F / \partial \beta=0$ we determine the magnetic fields $H_{c 1}$ and $H_{c 2}$. They fulfill the condition $M_{H}\left(H_{c 1}\right)=M_{H}\left(H_{c 2}\right)=0$, where $M_{H}=M_{\mathrm{FM}}$ $\cos (\theta-\beta)$ is the magnetization component of $M_{\mathrm{FM}}$ pointing parallel to the applied magnetic field (see Fig. 3). $M_{H}$ is the experimentally relevant FM magnetization component, which is measured by standard scalar magnetometry. In order to obtain explicit expressions for $H_{c 1}$ and $H_{c 2}$ we insert $\alpha=\alpha_{\text {eq }}, \beta_{1}\left(M_{H}=0\right)=\theta-\pi / 2$ and $\beta_{2}\left(M_{H}=0\right)=\theta-3 \pi / 2$ into $\partial F / \partial \beta=0$. Expansion of $\partial F / \partial \beta$ to first order with respect to $M_{\mathrm{AF}} \approx 0$ yields two corresponding linear equations in $H$, which provide $H_{c 1}$ and $H_{c 2}$, respectively. The exchange bias field is then calculated according to

$H_{\mathrm{e}}=\left(H_{c 1}+H_{c 2}\right) / 2$.

Although the calculation is straightforward, the result is lengthy. In order to simplify the resulting expression, $H_{\mathrm{e}}$ is again expanded into a Taylor series with respect to $M_{\mathrm{AF}} \approx 0$ and $1 / K_{\mathrm{AF}} \approx 0$ up to first and second order, respectively. The approximation of strong anisotropy, $1 / K_{\mathrm{AF}} \approx 0$, is consistent with the series expansion, Eq. (2). One 
finally obtains

$$
\begin{aligned}
\mu_{0} H_{\mathrm{e}}= & -\frac{J S_{\mathrm{AF}} S_{\mathrm{FM}} \cos \theta}{M_{\mathrm{FM}} t_{\mathrm{FM}}} \\
& -\left(\frac{J S_{\mathrm{AF}} S_{\mathrm{FM}} \cos \theta}{16 K_{\mathrm{AF}}^{2} M_{\mathrm{FM}}^{2} t_{\mathrm{AF}}^{2} t_{\mathrm{FM}}^{2}}\right. \\
& \times\left(-4 J K_{\mathrm{AF}} M_{\mathrm{AF}} S_{\mathrm{AF}} S_{\mathrm{FM}} t_{\mathrm{AF}}^{2}\right. \\
& +J^{2} M_{\mathrm{FM}} S_{\mathrm{AF}}^{2} S_{\mathrm{FM}}^{2} t_{\mathrm{FM}} \\
& +J K_{\mathrm{FM}} M_{\mathrm{AF}} S_{\mathrm{AF}} S_{\mathrm{FM}} t_{\mathrm{AF}} t_{\mathrm{FM}}-J S_{\mathrm{AF}} S_{\mathrm{FM}} \\
& \times\left(-4 J K_{\mathrm{AF}} M_{\mathrm{AF}} t_{\mathrm{AF}}^{2}+3 J M_{\mathrm{FM}} S_{\mathrm{AF}} S_{\mathrm{FM}} t_{\mathrm{FM}}\right. \\
& \left.+4 K_{\mathrm{FM}} M_{\mathrm{AF}} t_{\mathrm{AF}} t_{\mathrm{FM}}\right) \cos 2 \theta \\
& \left.\left.+3 J K_{\mathrm{FM}} M_{\mathrm{AF}} S_{\mathrm{AF}} S_{\mathrm{FM}} t_{\mathrm{AF}} t_{\mathrm{FM}} \cos 4 \theta\right)\right) .
\end{aligned}
$$

In the limit of infinite anisotropy $K_{\mathrm{AF}}$, Eq. (5) yields the $\theta$-dependent expression

$\mu_{0} H_{\mathrm{e}}=-\frac{J S_{\mathrm{AF}} S_{\mathrm{FM}} \cos \theta}{M_{\mathrm{FM}} t_{\mathrm{FM}}}$,

which has already been derived in Ref. [10]. In particular, Eq. (6) provides, again, the basic MB expression in the case $\theta=0$, which implies parallel orientation of the applied field with the easy axes.

The simplest possible $t_{\mathrm{AF}}$ dependence is derived from Eq. (5) in the limit $M_{\mathrm{AF}}=0$ and $\theta=0$ and finite, but strong anisotropy $K_{\mathrm{AF}}$. It reads

$\mu_{0} H_{\mathrm{e}}=-\frac{J S_{\mathrm{AF}} S_{\mathrm{FM}}}{M_{\mathrm{FM}} t_{\mathrm{FM}}}+\frac{J^{3} S_{\mathrm{AF}}^{3} S_{\mathrm{FM}}^{3}}{8 K_{\mathrm{AF}}^{2} M_{\mathrm{FM}} t_{\mathrm{FM}} t_{\mathrm{AF}}^{2}}$.

Eq. (7) qualitatively explains the steep increase and the subsequent saturation of $\left|\mu_{0} H_{\mathrm{e}}\right|$ with increasing AF layer thickness. Such behavior is not described within the alternative random field approach of Malozemoff [14], but has been reported by various authors [8,18,19]. While $\mathrm{Xi}$ and White [18] introduced a more complicated ansatz involving a helical structure of the AF magnetic moments, it is the aim of this analysis to stress the capabilities of the MB model.

The existence of a critical AF layer thickness was already pointed out by MB. It can simply be motivated from Eq. (3) on applying the condition $\alpha_{\text {eq }} \ll \pi$, as required within the scope of validity of
Eq. (2). In the limit $M_{\mathrm{AF}}=0$, Eq. (3) yields

$\alpha_{\mathrm{eq}}=\frac{\sin \beta}{\left(2 K_{\mathrm{AF}} t_{\mathrm{AF}} / J S_{\mathrm{AF}} S_{\mathrm{FM}}\right)+\cos \beta}$.

In order to prevent the unphysical divergence of $\alpha_{\text {eq }}$ for any direction of $M_{\mathrm{FM}}, 0 \leqslant \beta \leqslant \pi$, the condition $\left|K_{\mathrm{AF}} t_{\mathrm{AF}}\right|>\left|J S_{\mathrm{AF}} S_{\mathrm{FM}} / 2\right|$ has to be fulfilled and, hence, the existence of a critical $\mathrm{AF}$ layer thickness becomes obvious. Substitution of the conventional expression of the critical $\mathrm{AF}$ layer thickness $[2,3] t_{\mathrm{AF}}^{\mathrm{cr}}=\left|J S_{\mathrm{AF}} S_{\mathrm{FM}} / K_{\mathrm{AF}}\right|$ yields $\left|\alpha_{\text {eq }}\right|<1 / \sqrt{3}$ for $0 \leqslant \beta \leqslant \pi$, which proves that in fact $\alpha_{\text {eq }} \ll \pi$ is fulfilled for $t_{\mathrm{AF}}>t_{\mathrm{AF}}^{\mathrm{cr}}$.

Fig. 1 shows the best fit of Eq. (7) to the $\mu_{0} H_{\mathrm{e}}$ vs. $t_{\mathrm{AF}}$ data [8] of a $\mathrm{Ni}_{80} \mathrm{Fe}_{20}$ layer with thickness $t_{\mathrm{FM}}=6.5 \mathrm{~nm}$ deposited on top of FeMn for $3<t_{\mathrm{AF}}<12 \mathrm{~nm}$. The two-parameter fit yields $J S_{\mathrm{AF}} S_{\mathrm{FM}} /\left(M_{\mathrm{FM}} t_{\mathrm{FM}}\right)=0.013 \mathrm{~T}$ and $J^{3} S_{\mathrm{AF}}^{3} S_{\mathrm{FM}}^{3} /$ $\left(8 K_{\mathrm{AF}}^{2} M_{\mathrm{FM}} t_{\mathrm{FM}}\right)=1.08 \times 10^{-19} \mathrm{~T} / \mathrm{m}^{2}$. With $t_{\mathrm{FM}}=$ $6.5 \mathrm{~nm}$ and $M_{\mathrm{FM}}\left(\mathrm{Ni}_{80} \mathrm{Fe}_{20}\right)=0.73 \mathrm{MA} / \mathrm{m} \quad$ [20], we obtain the coupling energy $\left|J S_{\mathrm{AF}} S_{\mathrm{FM}}\right|=$ $6 \times 10^{-5} \mathrm{~J} / \mathrm{m}^{2}$ and the AF anisotropy $K_{\mathrm{AF}}=$ $7.3 \times 10^{3} \mathrm{~J} / \mathrm{m}^{3}$. The latter one is of the same order of magnitude as the $K_{\mathrm{AF}}$-values obtained, e.g., by Mathieu et al. [21] from Brillouin light scattering investigations and by Parkin et al. [12] from torque measurements. The above expression of the critical thickness then yields $t_{\mathrm{AF}}^{\mathrm{cr}}=8 \mathrm{~nm}$, which lies, however, 2.7 times above the steep increase of $\mu_{0} H_{\mathrm{e}}$ vs. $t_{\mathrm{AF}}$ shown in Fig. 1. Apparently, the situation can be improved by setting $t_{\mathrm{AF}}^{\mathrm{cr}}=J S_{\mathrm{AF}} S_{\mathrm{FM}} / 2 K_{\mathrm{AF}}$, which emerges as the lower bound of $t_{\mathrm{AF}}$ values fulfilling the condition $\left|K_{\mathrm{AF}} t_{\mathrm{AF}}\right|>\left|J S_{\mathrm{AF}} S_{\mathrm{FM}} / 2\right|$. In that case, the remaining error is reduced to less than $34 \%$. Note, however, that the inequality is only a necessary condition. It is not obvious, that its lower boundary can be identified with the critical thickness. This numerical discrepancy may originate from the strong simplifications, which underlay Eq. (7). One of them, the assumption $M_{\mathrm{AF}}=0$, will be discussed below.

Besides this numerical inconsistency (see above), the simple $1 / t_{\mathrm{AF}}^{2}$ dependence of Eq. (7) does not model any kind of a peak-like structure in the $\left|\mu_{0} H_{\mathrm{e}}\right|$ vs. $t_{\mathrm{AF}}$ dependence. This is, however, known from experiments, e.g., on $\mathrm{Ni}_{80} \mathrm{Fe}_{20} / \mathrm{FeMn}$ [8] or $\mathrm{Fe}_{3} \mathrm{O}_{4} / \mathrm{CoO}$ [22] bilayers as well as from 
Monte Carlo simulations [9]. In accordance with these findings, Eq. (5) exhibits the possibility of a competing $1 / t_{\mathrm{AF}}$-term in the case of $M_{\mathrm{AF}}>0$ and $\theta \neq 0$. The latter condition is not obvious, however, closer inspection of Eq. (5) shows that the $1 / t_{\mathrm{AF}^{-}}$ terms cancel each other in the case $\theta=0$. This is in full agreement with the results from Monte Carlo simulations on heterostructures of diluted antiferromagnets and FM layers [9]. As discussed above, the diluted antiferromagnet breaks into a random field domain state on cooling to below the Néel temperature in the presence of an applied magnetic field. These random field domains carry a frozen net magnetization $M_{\mathrm{AF}}$. Within the framework of the MB approach, Eq. (5), this opens the possibility for a $t_{\mathrm{AF}}$ dependence of the type

$\mu_{0} H_{\mathrm{e}}=a+\frac{b}{t_{\mathrm{AF}}}+\frac{c}{t_{\mathrm{AF}}^{2}}$.

Fig. 2 shows the result of a best fit of Eq. (9) to the Monte Carlo data of Ref. [9], which are obtained on a heterosystem with $40 \%$ of quenched dilution of the AF-sites (see Ref. [9] for details). The peak structure of $\left|\mu_{0} H_{\mathrm{e}}\right|$ vs. $t_{\mathrm{AF}}$ is qualitatively reproduced with fitting parameters $a=-9 \times 10^{-4}, b=-0.042$, and $c=0.037$ involving units adapted to the Monte Carlo data. Moreover, the simulations show that the peak strongly decreases for $30 \%$ as well as $60 \%$ dilution. This is reasonable, because the maximum frozen $\mathrm{AF}$ moment is expected to be induced by the maximum random field. In accordance with the approximation $h_{\mathrm{r}} \propto \sqrt{x(1-x)}$, the random field, $h_{\mathrm{r}}$, maximizes at $50 \%$ dilution, $x=0.5$ [23]. Obviously, both concentrations reduce the magnetic moment of the AF layer with respect to the case of $40 \%$ dilution. In agreement with the $\mathrm{MB}$ approach, Eq. (5), a reduction of $M_{\mathrm{AF}}$ gives rise to a reduced peak height. Hence, in accordance with the results of the Monte Carlo simulations our analysis suggests that apart from the random field enhanced AF interface magnetic moment $S_{\mathrm{AF}}$, the magnetization of the subsequent AF layers strongly influences the $\mu_{0} H_{\mathrm{e}}$ vs. $t_{\mathrm{AF}}$ behavior.

\section{Conclusion}

We present analytic descriptions of the exchange bias field of $\mathrm{FM} / \mathrm{AF}$ heterostructures based on a generalized Meiklejohn-Bean [2,3] ansatz of the free energy. They stress explicitly the dependencies on the thicknesses $t_{\mathrm{FM}}$ and $t_{\mathrm{AF}}$, on the orientation angle $\theta$ of the anisotropy axes with respect to the external field and on the frozen magnetization of the antiferromagnet, $M_{\mathrm{AF}}$. Within this framework, results from previous experimental investigations and simulations are successfully described. In particular $M_{\mathrm{AF}}$ turns out to be responsible for the observed peak structure in the $H_{\mathrm{e}}$ vs. $t_{\mathrm{AF}}$ dependence. While $M_{\mathrm{AF}}$ is a well-known feature of field-cooled dilute antiferromagnets, it seems to occur quite generally also in pure AF pinning substrates. This should be taken into account in future analytic investigations of the exchange bias which are beyond the MB approach.

\section{Acknowledgements}

Work supported by Deutsche Forschungsgemeinschaft within the framework of Sonderforschungs-bereich 491 ("Magnetische Heteroschichten").

\section{References}

[1] E.C. Stoner, E.P. Wohlfarth, Trans. R. Soc. (London) Ser. A 240 (1948) 599.

[2] W.H. Meiklejohn, C.P. Bean, Phys. Rev. 105 (1957) 904.

[3] W.H. Meiklejohn, J. Appl. Phys. 33 (1962) 1328.

[4] D. Mauri, E. Kay, D. Scholl, J.K. Howard, J. Appl. Phys. 62 (1987) 2929.

[5] X.W. Wu, C.L. Chien, Phys. Rev. Lett. 81 (1998) 2795.

[6] R.L. Stamps, J. Phys. D 33 (2000) R247.

[7] P. Miltenỳi, M. Gierlings, J. Keller, B. Beschoten, G. Güntherodt, U. Nowak, K.D. Usadel, Phys. Rev. Lett. 84 (2000) 4424.

[8] R. Jungblut, R. Coehoorn, M.T. Johnson, J. van de Stegge, R. Reinders, J. Appl. Phys. 75 (1994) 6659.

[9] U. Nowak, A. Misra, K.D. Usadel, J. Appl. Phys. 89 (2001) 7269.

[10] J.-V. Kim, R.L. Stamps, B.V. Mc Grath, R.E. Camley, Phys. Rev. B 61 (2000) 8888. 
[11] S. Riedling, M. Bauer, C. Mathieu, B. Hillebrands, R. Jungblut, J. Kohlhepp, R. Reinders, J. Appl. Phys. 85 (1999) 6648.

[12] S.S.P. Parkin, V. Speriosu, Magnetic properties of lowdimensional systems II, Proc. Phys. 50 (1990) 110.

[13] W. Kleemann, Int. J. Mod. Phys. B 7 (1993) 2469.

[14] A.P. Malozemoff, Phys. Rev. B 37 (1988) 7673.

[15] K. Takano, R.H. Kodama, A.E. Berkowitz, W. Cao, G. Thomas, Phys. Rev. Lett. 79 (1997) 1130.

[16] J. Nogués, C. Leighton, I.K. Schuller, Phys. Rev. B 61 (2000) 1315.

[17] B. Kagerer, Ch. Binek, W. Kleemann, J. Magn. Magn. Mater. 217 (2000) 139.
[18] H. Xi, R.M. White, Phys. Rev. B 61 (2000) 80.

[19] J. Nogués, I.K. Schuller, J. Magn. Magn. Mater. 192 (1999) 203.

[20] Ch. Kittel, Einführung in die Festkörperphysik, R. Oldenbourg, München, 1983.

[21] C. Mathieu, M. Bauer, B. Hillebrands, J. Fassbender, G. Güntherodt, R. Jungblut, J. Kohlhepp, A. Reinders, J. Appl. Phys. 83 (1998) 2863.

[22] P.J. van der Zaag, A.R. Ball, L.F. Feiner, R.M. Wolf, P.A.A. van der Heijden, J. Appl. Phys. 79 (1996) 5103.

[23] D.P. Belanger, A.P. Young, J. Magn. Magn. Mater. 100 (1991) 272. 